\title{
Non-pulmonary Rhodococcus equi infections in patients with acquired immune deficiency syndrome (AIDS)
}

\author{
J FIERER, P WOLF, LAURA SEED, T GAY, KATHLEEN NOONAN, P HAGHIGHI
}

From the Department of Medicine and Pathology, Veterans Administration Medical Center, San Diego, California, and the University of California, San Diego School of Medicine, USA

SUMMARY Rhodococcus equi, formerly known as Corynebacterium equi, was isolated repeatedly from the blood of two patients with the acquired immune deficiency syndrome (AIDS). Neither of ${ }_{0}$ the patients had pneumonia while they were bacteraemic, whereas pneumonia has been present incr all previously reported cases of human infection with $R$ equi. One of our patients had diarrhoea and $G$ the organism was isolated from a stool culture; the other patient had a large granulomatous soft $\mathrm{o}_{\circ}^{\circ}$ tissue mass in his pelvis caused by $R$ equi. Both isolates were resistant to penicillin and one produced? a $\beta$-lactamase. Both patients were treated with vancomycin but only one recovered.

Rhodococcus equi, previously known as Corynebacterium equi, is a well known pathogen of farm animals, but rarely causes disease in $\operatorname{man}^{1}$; only 15 cases of human infection have been reported, ${ }^{2-5}$ all but one of which were pulmonary infections in immunosuppressed patients. We report two further cases in patients with acquired immune deficiency syndrome (AIDS) complicated by $R$ equi bacteraemia. As far as we know these are the first reports of bacteraemia caused by extrapulmonary infection in man.

\section{Case reports}

CASE 1

A 35 year old white homosexual man had skin lesions of Kaposi's sarcoma with a history of Listeria monocytogenes bacteraemia and cryptosporidial gastroenteritis. He was admitted to the San Diego Veterans Administration for bloody diarrhoea and malnutrition. He had no respiratory symptoms and his chest $x$-ray was clear. Multiple blood cultures over two weeks grew $R$ equi, which was resistant to penicillin, cefazolin, and oxacillin but susceptible to vancomycin. Because of persistent diarrhoea, he had numerous stools examined with a modified acid fast stain. ${ }^{6}$ Two stool specimens showed lightly stained acid fast bacilli that did not resemble Mycobacterium . Coryneforme bacteria grew in pink colonies on the Lowenstein-Jensen's medium but were discarded

Accepted for publication 29 October 1986 without further identification. Mycobacterium avium- $\vec{\ominus}$ intracellularis also grew from the same stool speci- $-\infty$ mens. He was treated with vancomycin $(1 \mathrm{~g})$ daily for two weeks with no change in his symptoms. Threeo weeks later, just before his death, he complained of difficulty in breathing.

At necropsy there were numerous lesions of Kaposi's sarcoma and the lungs were consolidated $\stackrel{\mathbb{Q}}{\varrho}$ throughout with acute and chronic pneumonitis. $\overrightarrow{0}$ Microscopically there was an acute lobular pneu- 3 monitis with numerous microabscesses. No cultures were carried out. Adjacent areas showed the presence? of Pneumocystis carinii pneumonia. The retroperitoneal lymph nodes were necrotic, with destruc-? tion of the basic architecture, and were infiltrated by 3 . neutrophils, reactive lymphocytes, and macrophages with foamy cytoplasm. Brown and Brenn stains of $\frac{\rho}{3}$ lymph nodes showed many Gram positive bacilli which were pleomorphic and had coccoid forms. Acid 5 fast stains of retroperitoneal lymph nodes were posi- $\rightarrow$ tive for typical mycobacteria. No cultures were done.

CASE 2

A 55 year old bisexual man who had antibody to human immunodeficiency virus (HIV) developed severe back pain suggestive of a herniated lumbar disc. Computed tomography showed a soft tissue ${ }^{\circ}$ mass adjacent to the left pelvic sidewall, affecting the iliopsoas muscle, and extending out of the pelvis to the posterior aspect of the acetabulum and femoral neck (figure). An aspiration of the mass showed only fibrohistiocytic inflammation but $R$ equi was grown. $\frac{\stackrel{?}{\mathbb{D}}}{\mathrm{D}}$ A right paralumbar mass then developed. A biopsy 2 showed abnormally firm pale tissue beneath the deep? 
facia, which had replaced the normal muscle. Microscopic examination showed only chronic inflammation but Gram positive bacilli were seen and $R$ equi grew from the tissue. An enlarged axillary node was also removed. It showed a granulomatous infiltrate and grew both $R$ equi and Histoplasma capsulatum. During this admission three of four blood cultures grew $R$ equi. The blood culture isolates were resistant to penicillin $\mathrm{G}$ (minimum inhibitory concentration $=16 \mu \mathrm{g} / \mathrm{ml}$ ) and cephazolin but sensitive to gentamicin, erythromycin, and vancomycin. The culture was not tested for $\beta$-lactamase production. He was treated with amphotericin B and vancomycin for six weeks. The soft tissue masses gradually resolved. There was no clinical or radiographic evidence of pneumonia during this admission. He died at home from a wasting syndrome. Necropsy was not performed.

\section{Discussion}

$R$ equi was identified as a pathogen in 1923 when it was shown to be the cause of an enzootic pneumonia in foals. ${ }^{7}$ The principal pathology in foals was pneumonia and lung abscess with frequent suppuration in regional lymph nodes. Sometimes abscesses occur in other parts of the body, which are thought to be caused by haematogenous spread. ${ }^{1}$ Primary enteritis with mesenteric adenitis, however, has been reported in foals in the absence of pneumonia, suggesting that the portal of entry can be either the respiratory or the alimentary tract. $^{8}$ The natural habitat of the organism seems to be soil, which accounts for the prevalence of infection in grazing and rooting animals. About half the reported cases in man (seven of 15) had had contact with domestic animals. ${ }^{25}$ This may mean that $R$ equi is a zoonosis, or more likely, that people and their animals are exposed to contaminated soil. Neither of our patients had knowingly been exposed to animals.

Both of our cases presented with $R$ equi bacteraemia without pulmonary disease, which expands the spectrum of $R$ equi infection in man. ${ }^{2}$ Both patients were severely immunosuppressed because of HIV infection and they had multiple, often simultaneous, infections. Case 1 had coexisting $R$ equi and $M$ avium-intracellularis infection of the intestine and retroperitoneal nodes, and the alimentary tract may have been the source of his bacteremia. Interestingly, both $R$ equi and $M$ avium intracellularis are soil

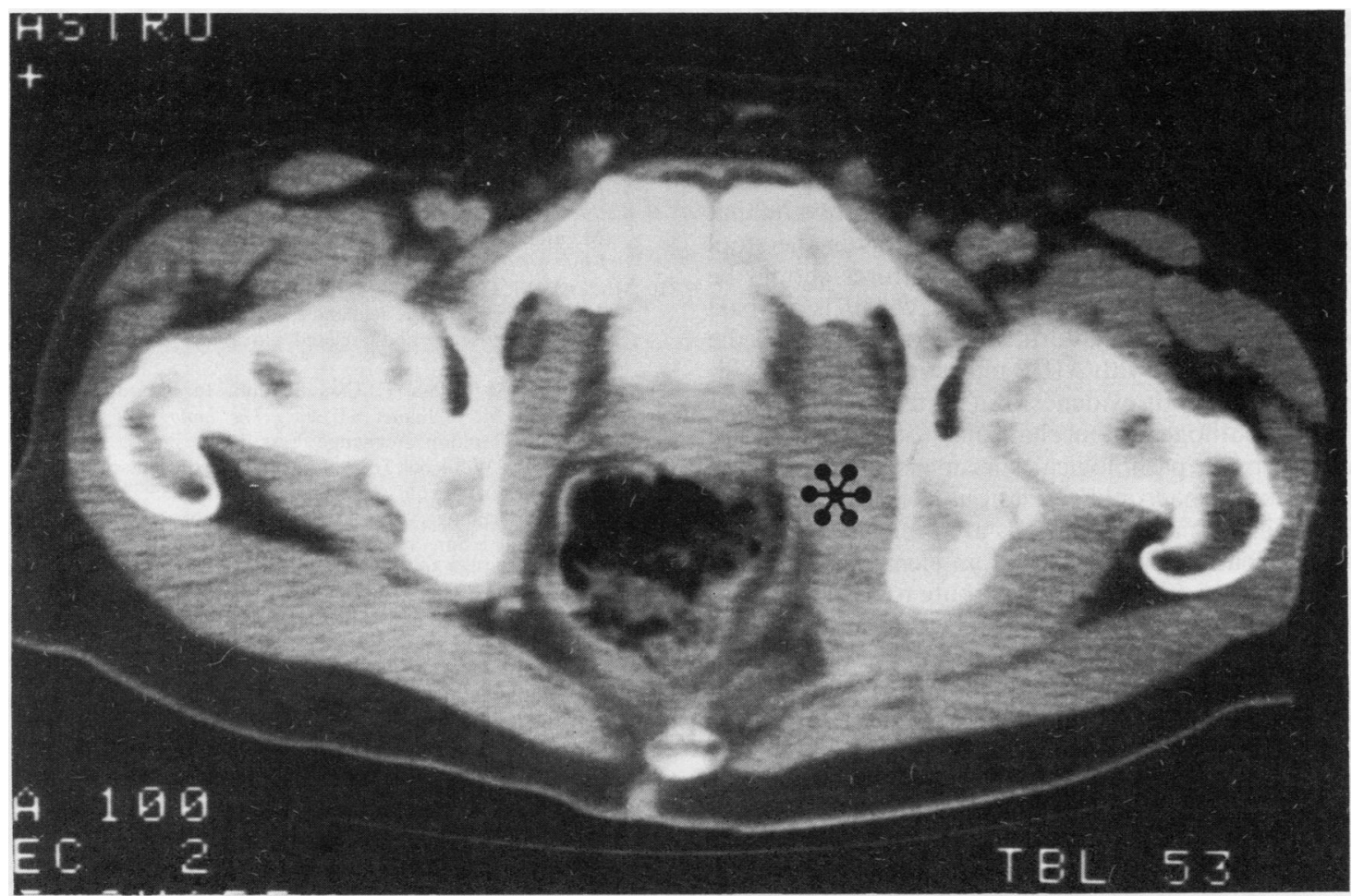

Figure Case 2: computed tomogram of pelvis at level of femoral heads before treatment. Star indicates large mass adjacent to iliac bone extending outside pelvis. 
organisms, and cervical adenitis in swine is often caused by mixed mycobacteria and $R$ equi infections. ${ }^{9}$ Case 2 had no obvious intestinal or pulmonary primary but presented with metastatic infection affecting retroperitoneal, pelvic, and axillary nodes. He had a psoas abscess by the time he presented, presumably from contiguous spread of infection from retroperitoneal nodes.

The taxomonic position of $R$ equi is still controversial. Goodfellow and Cross proposed assigning the organism to the genus Rodococcus. ${ }^{3}$ The organism is an actinomycete, having both mycolic acids and mesodiaminopamelic acid in its cell wall. It is also weakly acid fast and contains metachromatic granules. $R$ equi resembles Corynebacterium on Gram stain but the $\mathrm{G}+\mathrm{C} \%$ in $R$ equi DNA is close to $70 \%$ while true corynebacteria have a $\mathrm{G}+\mathrm{C} \%$ of less than "60\%". Furthermore, RNA-DNA hybridisation separates $R$ equi from other actinomycetes (Mycobacterium and Nocardium). ${ }^{10}$ Numerical classification also supports the species designation of $R$ equi. ${ }^{11}$

$R$ equi can be distinguished from corynebacteria by its mucoid, pale salmon-pink colonies and metabolic inactivity. It is non-fermentative and does not have any extracellular hydrolytic enzymes except urease, lipase, and alkaline phosphatase. ${ }^{12}$ If clinical laboratories are not aware that this organism is a potential human pathogen isolates from blood and wound cultures may be discarded as "diphtheroid" contaminants. Laboratories that are using modified acid fast stains to examine stools for Mycobacterium sp and Cryptosporidium sp, should also be aware that palely stained cocco-bacillary organisms in the stool may be $R$ equi, and appropriate cultures should be undertaken to isolate this organism. $R$ equi is not part of the normal faecal flora, and isolation from the stool of a patient with AIDS may stimulate the physician to search for evidence of a systemic infection.

The pathogenic mechanisms of $R$ equi are unknown. In pathological specimens organisms are found inside macrophages; lesions show mononuclear cell infiltrates; and old lesions are typically necrotic. ${ }^{1}$ This appearance suggests that an element of delayed type hypersensitivity may contribute to tissue destruction. The packed macrophages seen in our cases and in other cases of AIDS $^{45}$ resemble the histological appearance of $\boldsymbol{M}$ avium-intracellularis infections in patients with AIDS. ${ }^{13}$ It is likely that susceptibility of such patients to this organism is due to the inability to generate bactericidal macrophages, either because of depleted $\mathrm{T}$ helper lymphocytes or because of direct infection of the macrophage with HIV. ${ }^{14}$
The best treatment for $R$ equi infection in not yet known. Nearly all human isolates have been resistanto to penicillin, although some soil isolates are reportedly susceptible. ${ }^{15}$ The isolates from both of our cases were resistant to penicillin, and the isolate from case to produced a $\beta$ lactamase and hydrolysed nitrocephin. Isolates are uniformly susceptible to erythromycin, $\overline{\bar{\phi}}$ chloramphenicol, aminoglycosides, and vancomycin. $\mathbb{\otimes}$ Case 2 responded well to a long course of vancomycin. Relapses of infection are common, ${ }^{245}$ and $^{\infty}$ surgical excision of isolated lesions has been $\vec{\circ}$ recommended. ${ }^{2}$

\section{References}

1 Smith JE. Corynebacterium species as animal pathogens. J Appit Bacteriol 1966:29:119-30.

2 Van Etta L, Ferguson M, Gerding N. Corynebacterium equi: a review of 12 cases of human infection. Rev Infect Disç 1983;5:1012-18

3 Goodfellow M, Beckham AR, Barton MD. Numerical 을 classification of Rhodococcus equi and related actinomycetes. $\rightarrow$ J Appl Bacteriol 1982;53:199-207.

4 Samies JH, Hathaway BN, Echols RM, Veazey JM, Pilen VA. Lung abscess due to Corynebacterium equi: report of the first case in a patient with acquired immuno-deficiency syndrome. $\vec{c}$ Am J Med 1986;80:685-8.

5 Wong HH, Tollerud D, Donar D, Hanff P, Gottesdiener K, Rosen S. Another Whipple-like disease in AIDS? N Engl J Med 1986;314:1577-8.

6 Ma P, Soave R. Three-step stool examination for Cryptosporidiosis in 10 homosexual men with protracted watery diarrhoea. $J$ Infect Dis 1983;147:824-8.

7 Magnusson H. Pyemia in foals caused by Corynebacterium equi. Vet Rec 1938;50:1459-68.

8 Cimprich RE, Rooney JR. Corynebacterium equi enteritis in $\overline{\bar{B}}$ foals. Veterinary Pathology 977;14:95-102.

9 Karlson AG, Moses HE, Feldman WH. Corynebacterium equi. (Magnusson, 1923) in the submaxilliary lymph nodes of swine-0 J Infect Dis 1940;67:243-51.

10 Modarski M, Goodfellow M, Tkacz A, Pulverer G, Schaal KP. Ribosomal ribonucleic acid and similarities of the classification. of Rhodococcus and related taxa. $J$ Gen Microbio 1980;118:313-9.

11 Goodfellow M, Cross T. Classification. In: Goodfellow $M_{\Omega} \bigcirc$ Modarski M. Williams ST, eds. The biology of the acti? nomycetes. London: Academic Press, 1984.

12 Mutimer MD, Woolcock JB. A note on the hydrolytic enzymes of Corynebacterium equi. J Appl Bacteriol 1983;55:367-9.

13 Strom RL, Gruninget RS. AIDS with mycobacterium avium-O intracellular lesions resembling those of Whipple's disease. $N$ Engl J Med 1983;309:1323-4.

14 Ho DD, Rota TR, Hirsch MS. Infection of monocyte/ macrophages by human T lymphotropic virus type III. J Clin Invest 1986:77:1712-75

15 Woolcock JB, Mutimer MD. Corynebacterium equi in vitro susceptibility to twenty six antimicrobial agents. Antimicrob $<$ Agents Chemother 1980;18:976-7.

Requests for reprints to: Dr J Fierer, Professor of Medicine ${ }^{+}$ and Pathology, Veterans Administration Medical Center, San Diego, CA 92161, USA. 